\title{
Constraint algebra for Regge-Teitelboim formulation of gravity
}

\author{
S.A. Paston, A.N. Semenova ${ }^{\dagger}$ \\ Saint Petersburg State University, Russia
}

\begin{abstract}
We consider the formulation of the gravity theory first suggested by Regge and Teitelboim where the space-time is a four-dimensional surface in a flat ten-dimensional space. We investigate a canonical formalism for this theory following the approach suggested by Regge and Teitelboim. Under constructing the canonical formalism we impose additional constraints agreed with the equations of motion. We obtain the exact form of the first-class constraint algebra. We show that this algebra contains four constraints which form a subalgebra (the ideal), and if these constraints are fulfilled, the algebra becomes the constraint algebra of the Arnowitt-Deser-Misner formalism of Einstein's gravity. The reasons for the existence of additional first-class constraints in the canonical formalism are discussed.
\end{abstract}

*E-mail: paston@pobox.spbu.ru

${ }^{\dagger}$ E-mail: cat.86@mail.ru 


\section{Introduction}

More than 30 years ago, in 1975 T. Regge and C. Teitelboim suggested a formulation of gravity [1] similar to the formulation of string theory. They assumed that our space-time is a four-dimensional surface in ten-dimensional Minkowski space $R^{1,9}$ with one timelike and nine spacelike dimensions. In this case the variables describing the gravity are the embedding function of this surface into the ambient space. The authors take the standard Einstein-Hilbert expression for the action. In this expression they replace the metric by the induced metric expressed in terms of the embedding function. We will call this formulation of gravity the embedding theory. In this approach the equations of motion (the "Regge-Teitelboim equations") appear to be more general than the Einstein equations and they contain extra solutions.

To overcome the problem of extra solutions T. Regge and C. Teitelboim suggested in [1] to impose additional constraints $G_{\mu \perp}=0$ ("Einstein's constraints"), where $G_{\mu \nu}$ is the Einstein tensor, $\mu, \nu, \ldots=0,1,2,3$, and the symbol $\perp$ denotes the direction orthogonal to the constant time surface. While constructing the canonical formalism these constraints are considered similarly to the primary constrains, resulting in a system of eight constraints. We will call the theory arising from such approach the Regge-Teitelboim formulation of gravity, in contrast to the embedding theory.

The approach to the gravity based on the consideration of a surface in a flat Minkowski space could be more convenient than a standard approach when we try to develop a quantum theory of gravity, since in this case we have a possibility to formulate the causality principle more clearly. In the quantum field theory the causality principle usually means the commutativity of operators related to areas separated by a spacelike interval. This principle is difficult to formulate in the framework of the standard gravity formulation in terms of metric $g_{\mu \nu}$, because the interval between points is determined by the metric which is an operator itself. Therefore for two specific points of the space-time it is impossible to determine what kind of interval separates them independently of a specific state. In the case of the description of gravity as a dynamics of a three-dimensional surface in a flat ambient space, we can try to work out a quantum field theory giving this gravity in the classical limit. In this case the problem of formulation of the causality principle would be solved, since the causality in the flat ambient space can be determined by standard means of quantum field theory.

The Regge-Teitelboim formulation of gravity has been discussed in the work [2] published immediately after [1. The authors remarked that the Regge-Teitelboim equations are trilinear in the second derivatives of the embedding function. This fact is very significant for this approach as it obstructs linearizing the theory near the flat surface. Also in [2] the problem of absence of uniqueness of the embedding is discussed. The lack of uniqueness causes a question whether the transfer from one surface to another with the same metric corresponds to the change of some physical degrees of freedom, or such a transfer should be considered as "a change of the embedding gauge". The discussion of these problems is beyond the scope of our article.

In the paper [2] it is also noted that an artificial, ad hoc, imposing of additional constraints to the theory seems not quite satisfactory and that a more satisfactory alternative would be to find another action whose Euler-Lagrange equation would be equivalent to Einstein equations. Such an action was suggested in the paper [10]. In Section 4 of our paper we clarify the way of building the action and discuss the meaning of the existence of additional first-class constraints in the canonical formalism. 
After the articles [1, 2] the idea of embedding was used for description of gravity quite often. In particular, the canonical formalism for the embedding theory without imposing Einstein's constraints was investigated in [3, 4, 5]. Such a canonical formalism turns out to be very complicated. Among recent works using the idea of embedding we mark [6, 7, 8]. An extended bibliography related to the embedding theory and similar problems can be found in [9].

In the work [1] the form of the constraints system for Regge-Teitelboim formulation of gravity was found. Also the problem has been formulated to investigate the algebra of these constraints and to verify whether these constraints are the first-class constraints. However this problem is not completely solved by now. It is probably due to the fact that one of constraints in [1] was written incorrectly, as it was shown in [10], see details in Sec. 2.

We started to work on this problem in the article [11]. It was analyzed in detail under what conditions the imposition of Einstein's constraints turns the Regge-Teitelboim equations into the Einstein's equations. This is true in generic case, i. e., except some special values of variables at a fixed instant. The canonical formalism for Regge-Teitelboim formulation of gravity was built anew in [10] with a supplementary imposition of Einstein's constraints. A correct form of all constraints was obtained, however the constraint algebra has not been found completely. In this paper we are completing the solution of the problem. We perform an accurate calculation of the Poisson brackets between constraints and, as a result, we obtain a first-class constraint algebra for Regge-Teitelboim formulation of gravity.

For convenience we describe in Section 2 the construction of the canonical formalism for Regge-Teitelboim formulation of gravity following [10]. We do it in particular in order to explain why we regard that in [1] one of constraints was written incorrectly and to show how we obtain a correct form of all constraints.

Section 3 contains the main result of this paper. In this section we find all Poisson brackets between constraints and we obtain the first-class constraint algebra. We also discuss the relation between this algebra and the constraint algebra of Arnowitt-Deser-Misner formalism for the Einstein's gravity. The formalism used in calculations is described in [10, 11].

\section{Canonical formalism with additionally imposed Einstein's constraints}

In this section we build a canonical formalism for Regge-Teitelboim formulation of gravity following [10]. We additionally impose Einstein's constraints as it was suggested in [1].

The embedding function determining the four-dimensional surface $W^{4}$ in the flat tendimensional space $R^{1,9}$ is the map

$$
y^{a}\left(x^{\mu}\right): R^{4} \longrightarrow R^{1,9} .
$$

Here and below, the indices $a, b, \ldots$ run over the values $0,1,2, \ldots, 9$; and $y^{a}$ are the Lorentzian coordinates in $R^{1,9}$. A constant metric $\eta_{a b}=\operatorname{diag}(1,-1,-1, \ldots,-1)$ in the ambient space $R^{1,9}$ can easily rise and lower indices. It induces on the surface $W^{4}$ the metric

$$
g_{\mu \nu}=\eta_{a b} \partial_{\mu} y^{a} \partial_{\nu} y^{b}=\eta_{a b} e_{\mu}^{a} e_{\nu}^{b}
$$

where $e_{\mu}^{a} \equiv \partial_{\mu} y^{a}$.

We take the theory action in the form of the standard Einstein-Hilbert expression

$$
S=\int d^{4} x \sqrt{-g} R
$$


where we substitute the induced metric expressed in terms of the embedding function $y^{a}(x)$ by formula (2). We consider the gravity with matter absent, because adding matter does not play a fundamental role in the analysis of the theory.

Note that the issue of changing the physical content of the theory under a non-point change of variables including time derivatives was studied in paper [12. It has been shown, under some assumptions, that if after the change of variables the higher derivatives are contained in the Lagrangian only in the form of a combination being a total time derivative, then the physical content of the theory remains unchanged. Substituting (2) in the action (3), the above condition is fulfilled (see below). Nevertheless in this case the result [12] is inapplicable, as the assumptions made there are violated. In particular, the change of variables (2) is quadratic whereas in the paper [12] only infinitesimal transformations are considered.

Varying action (3) with respect to $y^{a}(x)$ gives the Regge-Teitelboim equations which can be written as

$$
G^{\mu \nu} b_{\mu \nu}^{a}=0
$$

where $G^{\mu \nu}$ is Einstein's tensor, and

$$
b_{\mu \nu}^{a}=\prod_{\perp b}^{a} \partial_{\mu} \partial_{\nu} y^{b}=\nabla_{\mu} e_{\nu}^{a}
$$

is the second fundamental form of the surface. Here $\nabla_{\mu}$ is the covariant derivative, and the quantity $\Pi_{\perp b}^{a}$ is the projector on the space orthogonal to the surface $W^{4}$ at a given point. We note that although the free index a ranges over 10 values, there are only 6 independent equations, and the rest 4 equations satisfy identically because of the properties of the second fundamental form of the surface.

Besides the solutions of Einstein equations $G^{\mu \nu}=0$, the equations (44) contain extra solutions which can be excluded (in general case) by imposing at the initial instant the Einstein's constraints

$$
n_{\mu} G^{\mu \nu}=0
$$

where $n_{\mu}$ is a unit vector normal to surfaces $x^{0}=$ const at each point (see [10, 11]).

For developing a canonical formalism it is convenient to drop the total derivative term in the integrand in (3) and to rewrite the action under the Arnowitt-Deser-Misner (ADM) form [13]:

$$
S=\int d^{4} x \sqrt{-g}\left(\left(K_{i}^{i}\right)^{2}-K_{i k} K^{i k}+\stackrel{3}{R}\right),
$$

where $K_{i k}$ is the second fundamental form of the surface $t=$ const considered as a submanifold in $W^{4}$. Here and below, the indices $i, k, \ldots$ range the values $1,2,3$, and we label the quantities related to the surface $t=$ const with the digit " 3 " over the letter.

If we rewrite this expression in terms of the embedding function $y^{a}(x)$ it becomes

$$
S=\int d^{4} x \sqrt{-g}\left(n_{a} n_{b} \stackrel{3}{b}_{i k}^{a} \stackrel{3}{b}_{l m}^{b} L^{i k, l m}+\stackrel{3}{R}\right)
$$

where we introduced

$$
L^{i k, l m}=\stackrel{3}{g}^{i k} \stackrel{3}{g} l m-\frac{1}{2}\left(\stackrel{3}{g}^{i l} \stackrel{3}{g} k m+\stackrel{3}{g}^{i m} \stackrel{3}{g}^{k l}\right), \quad L^{i k, l m}=L^{k i, l m}=L^{l m, i k}
$$


(this quantity is equal to a known Wheeler-De Witt metric within a factor). The action (8) can be rewritten in the form where the derivatives $\dot{y}^{a} \equiv \partial_{0} y^{a}$ of the variables $y^{a}(x)$ with respect to the time $x^{0}$ are written explicitly:

$$
\begin{gathered}
S=\int d x^{0} L\left(y^{a}, \dot{y}^{a}\right), \\
L=\int d^{3} x \frac{1}{2}\left(\frac{\dot{y}^{a} B_{a b} \dot{y}^{b}}{\sqrt{\dot{y}^{a} \prod_{\perp a b}^{3} \dot{y}^{b}}}+\sqrt{\dot{y}^{a} \prod_{\perp a b}^{3} \dot{y}^{b}} B_{c}^{c}\right),
\end{gathered}
$$

where the quantity

$$
B^{a b}=2 \sqrt{-\stackrel{3}{g}} \stackrel{3}{b}_{i k}^{a} \stackrel{3}{b}{ }_{l m}^{b} L^{i k, l m}
$$

as well as the projection operator $\prod_{\perp a b}^{3}$ do not contain time derivatives.

We find the generalized momentum $\pi_{a}$ for the variable $y^{a}$ from action (10):

$$
\pi_{a}=\frac{\delta L}{\delta \dot{y}^{a}}=B_{a b} n^{b}-\frac{1}{2} n_{a}\left(n_{c} B^{c d} n_{d}-B_{c}^{c}\right),
$$

where we use the formula

$$
n^{a}=\frac{{\stackrel{3}{\prod_{\perp}} a}_{b}^{a} \dot{y}^{b}}{\sqrt{\dot{y}^{c} \prod_{\perp c d}^{3} \dot{y}^{d}}} .
$$

We suppose that besides the primary constraints appearing from this equality, four Einstein's constraints (6) are also satisfied. As shown in [10, 11], they can be written as

$$
\begin{gathered}
\mathcal{H}^{0}=\frac{1}{2}\left(n_{c} B^{c d} n_{d}-B_{c}^{c}\right) \approx 0, \\
\mathcal{H}^{i}=-2 \sqrt{-\stackrel{3}{g}} \stackrel{3}{\nabla}_{k}\left(L^{i k, l m} \stackrel{3}{b}_{l m}^{a} n_{a}\right) \approx 0 .
\end{gathered}
$$

We note that the definitions of the constraints (14) differ from these used in [10] by the factor $1 / 2$.

If we use the constraint (14) in equality (12), then it takes a simple form

$$
\pi_{a}=B_{a b} n^{b} .
$$

Taking account of (11) and of the properties of the quantity $\stackrel{3}{b}_{i k}^{a}$, we immediately obtain three primary constraints

$$
\Phi_{i}=\pi_{a} \stackrel{3}{a}_{i}^{a} \approx 0
$$

One more constraint, the fourth one, has to appear. In [1] it was obtained from the unit vector $n^{b}$ normalization under the form

$$
\left(B^{-1} \pi\right)^{2}-1 \approx 0
$$


where $B^{-1}$ meaned the inversion of matrix $B$ in seven-dimensional subspace normal to the surface $W^{3}$. However, this form was incorrect, because the matrix $B$ has rank 6 in general case and could not be inverted in the seven-dimensional subspace mentioned above.

Indeed, the quantity $\stackrel{3}{b}_{i k}^{a}$ can be considered as a set of six vectors (at fixed values of indices $i, k$ over which it is symmetric). On the other hand, this quantity satisfies three identities $\stackrel{3}{b}_{i k}^{a} \stackrel{3}{e}_{a, l}=0$. Therefore, in general case there exists a unique vector $w_{a}$ determined by conditions

$$
w_{a} \stackrel{3}{a}_{l}^{a}=0, \quad w_{a} \stackrel{3}{b}_{i k}^{a}=0, \quad\left|w_{a} w^{a}\right|=1
$$

The matrix $B^{a b}$ gives a zero when it acts on this vector lying in the seven-dimensional subspace mentioned above. Hence, it can not be inverted in this subspace. Instead of (18) the fourth primary constraint has to be written as

$$
\Psi^{4}=\pi_{a} w^{a} \approx 0
$$

(the reason for such notation will be obvious below) and the condition of normalization of vector $n^{b}$ does not lead to new limitations.

Using formulas (10), (13), (16), one easily founds that the Hamiltonian of the theory

$$
H=\int d^{3} x \pi_{a} \dot{y}^{a}-L
$$

vanishes. Therefore, the generalized Hamiltonian reduces to a linear combination of constraints (14), (15), (17), (20) .

In the canonical formalism, constraints must be expressed via generalized coordinates and momenta, i. e., via $y^{a}$ and $\pi_{a}$ but not $\dot{y}^{a}$ in our case. Constraints (17) and (20) satisfy this requirement (we note that vector $w_{a}$ determined by conditions (19) depends on $y^{a}$, but not on $\dot{y}^{a}$ ), while constraints (14) and (15) do not satisfy it. They must therefore be transformed to the necessary form. For this, we introduce the quantity $\alpha_{a}^{i k}$ unambiguously determined by the conditions

$$
\alpha_{a}^{i k}=\alpha_{a}^{k i}, \quad \alpha_{a}^{i k} \stackrel{3}{e}_{l}^{a}=0, \quad \alpha_{a}^{i k} w^{a}=0, \quad \alpha_{a}^{i k} \stackrel{3}{b}_{l m}^{a}=\frac{1}{2}\left(\delta_{l}^{i} \delta_{m}^{k}+\delta_{m}^{i} \delta_{l}^{k}\right) .
$$

The value $\alpha_{a}^{i k}$ can be considered as inverse to $\stackrel{3}{b}_{l m}^{a}$, and

$$
\alpha_{b}^{i k} \stackrel{3}{b}{ }_{i k}^{a}=\prod_{\perp}^{3} \underset{b}{a}-\frac{w^{a} w_{b}}{w^{c} w_{c}}
$$

where the right part contains the projector on the six-dimensional subspace normal to the surface $W^{3}$ and to the vector $w^{a}$.

It is clear that $\alpha_{a}^{i k}$ as well as $w_{a}$ depends on $y^{a}$ but not on $\dot{y}^{a}$. Relation (16) implies that

$$
\stackrel{3}{b}_{i k}^{b} n_{b}=\frac{1}{2 \sqrt{-\stackrel{3}{g}^{3}}} \hat{L}_{i k, l m} \alpha_{a}^{l m} \pi^{a},
$$

where

$$
\begin{gathered}
\hat{L}_{p r, l m}=\frac{1}{2}\left(g_{p r} g_{l m}-g_{p l} g_{r m}-g_{p m} g_{r l}\right), \\
\hat{L}_{p r, l m} L^{i k, l m}=\frac{1}{2}\left(\delta_{p}^{i} \delta_{r}^{k}+\delta_{r}^{i} \delta_{p}^{k}\right) .
\end{gathered}
$$


Using formula (24), the constraints (14), (15) can be expressed in terms of $y^{a}$ and $\pi_{a}$. It is convenient to use a linear combination $\Psi^{i}=\mathcal{H}^{i}+\stackrel{3}{g}^{i k} \Phi_{k}$ instead of the constraint $\mathcal{H}^{i}$. As a result, we have a set of eight constraints:

$$
\begin{aligned}
\Phi_{i}=\pi_{a} \stackrel{3}{e}_{i}^{a}, \quad \Psi^{i} & =-\sqrt{-\stackrel{3}{g}} \stackrel{3}{\nabla}_{k}\left(\frac{1}{\sqrt{-\stackrel{3}{g}}} \pi^{a} \alpha_{a}^{i k}\right)+\pi^{a} \stackrel{3}{e}_{a}^{i}, \quad \Psi^{4}=\pi_{a} w^{a}, \\
\mathcal{H}^{0} & =\frac{1}{4 \sqrt{-\stackrel{3}{g}}} \pi^{a} \alpha_{a}^{i k} \hat{L}_{i k, l m} \alpha_{b}^{l m} \pi^{b}-\sqrt{-\stackrel{3}{g}} \stackrel{3}{R} .
\end{aligned}
$$

We can see that all constraints except $\mathcal{H}^{0}$ are linear in momentum $\pi^{a}$, and the constraint $\mathcal{H}^{0}$ is quadratic.

\section{Constraint algebra}

In this section we find the exact form of all Poisson brackets between the constraints. It will be seen that these Poisson brackets are linear combinations of the constraints, therefore this set of eight constraints forms a first-class constraint algebra for Regge-Teitelboim formulation of gravity. We drop some tedious algebraic transformations using formulas described in [10, 11].

It is convenient to work with constraints convoluted with arbitrary functions. It is also convenient to join constraints $\Psi^{i}$ and $\Psi^{4}$ under the index $A$ ranging the values $1,2,3,4$, since, as it will be shown, their action on variables has similar geometrical meaning in spite of their different nature $\left(\Psi^{4}\right.$ is a primary constraint and $\Psi^{i}$ contains an additionally imposed constraint $\left.\mathcal{H}^{i}\right)$. We use denotations

$$
\begin{gathered}
\Phi_{\xi} \equiv \int d^{3} x \Phi_{i}(x) \xi^{i}(x)=\int d^{3} x \pi_{a} \stackrel{3}{a}_{i}^{a} \xi^{i}, \quad \mathcal{H}_{\xi}^{0} \equiv \int d^{3} x \mathcal{H}^{0}(x) \xi(x), \\
\Psi_{\xi} \equiv \int d^{3} x \Psi^{A}(x) \xi_{A}(x)=\int d^{3} x \pi^{a}\left(\alpha_{a}^{i k} \stackrel{3}{\nabla}_{i} \xi_{k}+\stackrel{3}{e}_{a}^{k} \xi_{k}+w_{a} \xi_{4}\right)= \\
=\int d^{3} x \pi^{a} V_{a}^{A} \xi_{A},
\end{gathered}
$$

where a denotation for the differential operator is used:

$$
V_{a}^{i}=\alpha_{a}^{i k} \stackrel{3}{\nabla}_{i}+\stackrel{3}{e}_{a}^{k}, \quad V_{a}^{4}=w_{a}
$$

First of all, we find a geometrical meaning of three constraints $\Phi_{i}$. For this purpose we calculate their action on variables. It is easy to find that

$$
\left\{\Phi_{\xi}, y^{a}(x)\right\}=\xi^{i}(x) \partial_{i} y^{a}(x), \quad\left\{\Phi_{\xi}, \frac{\pi_{a}(x)}{\sqrt{-\stackrel{3}{g}^{3}(x)}}\right\}=\xi^{i}(x) \partial_{i} \frac{\pi_{a}(x)}{\sqrt{-\stackrel{3}{g}(x)^{3}}},
$$

where $\{\ldots\}$ is a Poisson bracket. It means that $\Phi_{\xi}$ generates a transformation $x^{i} \rightarrow x^{i}+\xi^{i}(x)$ of three-dimensional coordinates on the constant-time surface $W^{3}$ (it should be noted that generalized momentum $\pi^{a}$ is a three-dimensional scalar density). Because all constraints (27) are covariant (in three-dimensional meaning) equalities, we can write the action of constraints $\Phi_{i}$ on them:

$$
\left\{\Phi_{\xi}, \Phi_{\zeta}\right\}=-\int d^{3} x \Phi_{k}\left(\xi^{i} \stackrel{3}{\nabla}_{i} \zeta^{k}-\zeta^{i} \stackrel{3}{\nabla}_{i} \xi^{k}\right)
$$




$$
\begin{gathered}
\left\{\Phi_{\xi}, \Psi_{\zeta}\right\}=-\int d^{3} x\left(\Psi^{k}\left(\xi^{i} \stackrel{3}{\nabla}_{i} \zeta_{k}+\zeta_{i} \stackrel{3}{\nabla}_{k} \xi^{i}\right)+\Psi^{4} \xi^{i} \partial_{i} \zeta_{4}\right) \\
\left\{\Phi_{\xi}, \mathcal{H}_{\zeta}^{0}\right\}=-\int d^{3} x \mathcal{H}^{0} \xi^{i} \partial_{i} \zeta
\end{gathered}
$$

Now we find a geometrical meaning of four constraints $\Psi^{A}$. It is easy to verify that

$$
\left\{\Psi_{\xi}, \stackrel{3}{g}_{i k}(x)\right\}=0
$$

so the constraints $\Psi^{A}$ generate transformations which are an isometric bending of the surface $W^{3}$ (we stress that it is true as for $\Psi^{i}$ so for $\Psi^{4}$ ). We note that the number (four) of the found generators of three-dimensional isometric bendings corresponds to the difference between the dimensionality (ten) of the space into which the three-dimensional surface is embedded and the number of independent components (six) of the three-dimensional metric.

It is useful to calculate the action of constraints $\Psi^{A}$ on quantity

$$
\pi^{l m} \equiv-\pi^{a} \alpha_{a}^{l m} / 2
$$

The calculation gives a rather long equality where each term is proportional to one of constraints $\Psi^{A}$. Thus, under the action of $\Psi^{A}$ the quantity $\pi^{l m}$ does not change if $\Psi^{A}=0$. Since $\mathcal{H}^{0}$ and $\mathcal{H}^{i}$ can be expressed by quantities $\stackrel{3}{g}_{l m}$ and $\pi^{l m}$ (see (26) ), we can at once conclude (taking in to account (31)) that the Poisson bracket of constraint $\Psi_{\xi}$ with constraints $\Psi^{i}$ and $\mathcal{H}^{0}$ reduces to a linear combination of constraints. After tedious calculations we obtain an exact result of the action of constraints $\Psi^{A}$ on other constraints:

$$
\begin{gathered}
\left\{\Psi_{\xi}, \Psi_{\zeta}\right\}=\int d^{3} x\left(\delta y_{\Psi_{\xi}}^{a} \bar{\Psi}_{a b} \delta y_{\Psi_{\zeta}}^{b}-\delta y_{\Psi_{\zeta}}^{a} \bar{\Psi}_{a b} \delta y_{\Psi_{\xi}}^{b}\right) \\
\left\{\Psi_{\xi}, \mathcal{H}_{\zeta}^{0}\right\}=\int d^{3} x\left(\delta y_{\Psi_{\xi}}^{a} \bar{\Psi}_{a b} \delta y_{\mathcal{H}_{\zeta}^{0}}^{b}-\delta y_{\mathcal{H}_{\zeta}^{0}}^{a} \bar{\Psi}_{a b} \delta y_{\Psi_{\xi}}^{b}\right),
\end{gathered}
$$

where the quantity

$$
\bar{\Psi}_{a b}=\left(\Psi^{i} \eta_{a b}-\Psi^{4} \frac{w_{b}}{w_{c} w^{c}} V_{a}^{i}\right) \stackrel{3}{\nabla}_{i}
$$

is a linear combination of the constraints $\Psi^{A}$, being also (like $V_{a}^{A}$, see (28)) a differential operator. We have denoted

$$
\delta y_{\Psi_{\xi}}^{a}(x)=\left\{\Psi_{\xi}, y^{a}(x)\right\}=V^{a A} \xi_{A}(x), \quad \delta y_{\mathcal{H}_{\zeta}^{0}}^{a}(x)=\left\{\mathcal{H}_{\zeta}^{0}, y^{a}(x)\right\}=\hat{B}^{a c} \pi_{c} \zeta
$$

for the results of acting of constraints on the independent variable $y^{a}(x)$, where

$$
\hat{B}^{a c}=\frac{1}{2 \sqrt{-g}} \alpha_{i k}^{a} \alpha_{l m}^{c} \hat{L}^{i k, l m}
$$


is the inverted quantity to $B_{c b}$ in six-dimensional subspace normal to the surface $W^{3}$ and to the vector $w^{a}$ :

$$
\hat{B}^{a c} B_{c b}=\prod_{\perp}^{3} a b-\frac{w^{a} w_{b}}{w^{c} w_{c}}
$$

(formulas (22), (23), (25) are used).

In order to complete finding of the full constraint algebra we need to calculate the Poisson bracket of the constraint $\mathcal{H}^{0}$ with itself. This calculation is the most tedious and gives:

$$
\left\{\mathcal{H}_{\xi}^{0}, \mathcal{H}_{\zeta}^{0}\right\}=\int d^{3} x\left(\delta y_{\mathcal{H}_{\xi}^{0}}^{a} \bar{\Psi}_{a b} \delta y_{\mathcal{H}_{\zeta}^{0}}^{b}-\delta y_{\mathcal{H}_{\zeta}^{0}}^{a} \bar{\Psi}_{a b} \delta y_{\mathcal{H}_{\xi}^{0}}^{b}-\left(\Psi^{k}-\stackrel{3}{g} k l \Phi_{l}\right)\left(\xi \stackrel{3}{\nabla_{k} \zeta}-\zeta \stackrel{3}{\nabla}_{k} \xi\right)\right)
$$

The formulas (30)-(31), (35), (36), (41) gives the exact form of the first-class constraint algebra for Regge-Teitelboim formulation of gravity. It should be noted that the results of calculation of Poisson brackets (35), (36) and partially (41) have similar structure. The reason for that is unclear.

According to what is written after the formula (21), the generalized Hamiltonian of the theory can be written in the form

$$
H^{\text {gen }}=\int d^{3} x\left(\tilde{\lambda}^{i} \Phi_{i}+N_{A} \Psi^{A}+N_{0} \mathcal{H}^{0}\right)
$$

As can be seen from (35) (taking into account (37)), the four constraints $\Psi^{A}$ generating the isometric bending of the surface $W^{3}$ form a subalgebra in the full constraint algebra. It means that the Poisson brackets between them are reduced to their linear combination. Moreover, it is seen that the Poisson brackets of the constraints $\Psi^{A}$ with all other constraints (and consequently with the Hamiltonian (42)) also reduce to such a linear combination. Thus the constraints $\Psi^{A}$ form the ideal. It means that once imposed the constraints $\Psi^{A}$ remain satisfied in the time independently of satisfying other constraints.

If we limit our consideration of the system with satisfied constraints $\Psi^{A}=0$, then its dynamics will be determined by the Hamiltonian

$$
\begin{aligned}
\tilde{H}=\int d^{3} x\left(\tilde{\lambda}^{i} \Phi_{i}\right. & \left.+N_{0} \mathcal{H}^{0}\right)=\int d^{3} x\left(-\tilde{\lambda}_{i} \mathcal{H}^{i}+N_{0} \mathcal{H}^{0}\right)= \\
& =\int d^{3} x\left(-2 \tilde{\lambda}_{i} \sqrt{-\stackrel{3}{g}^{*}} \stackrel{3}{\nabla}_{k}\left(\frac{\pi^{i k}}{\sqrt{-\stackrel{3}{g}}}\right)+N_{0}\left(\frac{\pi^{i k} \hat{L}_{i k, l m} \pi^{l m}}{\sqrt{-\stackrel{3}{g}}}-\sqrt{-\stackrel{3}{g}} \stackrel{3}{R}\right)\right)
\end{aligned}
$$

where $\Phi_{i}$ was expressed by $\mathcal{H}^{i}$, the formulas (26) were applied, and the quantity $\pi^{i k}$ determined by (34) was used. This Hamiltonian as a functional of the quantities $\stackrel{3}{g}_{i k}$ and $\pi^{i k}$ coincides exactly with the known Hamiltonian expression in the ADM formalism. Besides, it is easy to verify that these quantities $\stackrel{3}{g}_{i k}$ and $\pi^{i k}$ are canonically conjugate at $\Psi^{A}=0$ (it should be noted that this condition is necessary only for vanishing of the Poisson bracket $\left.\left\{\pi^{i k}(x), \pi^{l m}(\tilde{x})\right\}\right)$. Therefore the dynamics of Regge-Teitelboim formulation of gravity on the surface of constraints $\Psi^{A}=0$ coincides with the dynamics of gravity in the ADM formalism. 


\section{Discussion about existence of additional first-class constraints}

In this section we discuss what could mean the existence in the canonical formalism of additional constraints which are in involution with the Hamiltonian of the theory and which form a first-class constraint algebra, probably with other constraints inherent in the theory. The Einstein's constraints (14), (15) for Regge-Teitelboim formulation of gravity are just these additional constraints.

For comparison we consider a simple model in the Minkowski space with the action

$$
S=\int d t \int d^{3} x\left(\frac{1}{2}\left(\partial_{0} A_{i}\right)\left(\partial_{0} A_{i}\right)-\frac{1}{4}\left(\partial_{i} A_{k}-\partial_{k} A_{i}\right)\left(\partial_{i} A_{k}-\partial_{k} A_{i}\right)\right),
$$

where the independent variable is a three-component field $A_{i}(x)$. The generalized momentum is the quantity $\pi_{i}=\partial_{0} A_{i}$, the primary constraints are absent. The Hamiltonian has form

$$
H=\int d^{3} x\left(\frac{1}{2} \pi_{i} \pi_{i}+\frac{1}{4}\left(\partial_{i} A_{k}-\partial_{k} A_{i}\right)\left(\partial_{i} A_{k}-\partial_{k} A_{i}\right)\right) .
$$

We consider an additional constraint $\Phi(x)=\partial_{i} \pi_{i}(x)$. It is easy to verify that it is in involution with the Hamiltonian, so their Poisson bracket $\{H, \Phi(x)\}=0$ vanishes. Because of $\{\Phi(x), \Phi(y)\}=0$, the quantity $\Phi(x)$ is the first-class constraint and can be added to the Hamiltonian with a Lagrange factor:

$$
H^{\text {gen }}=\int d^{3} x\left(\frac{1}{2} \pi_{i} \pi_{i}+\frac{1}{4}\left(\partial_{i} A_{k}-\partial_{k} A_{i}\right)\left(\partial_{i} A_{k}-\partial_{k} A_{i}\right)+\lambda \partial_{i} \pi_{i}\right) .
$$

Therefore, the case of the additional imposed constraint $\Phi(x)$ in this model is completely analogous to the case of Einstein's constraints in Regge-Teitelboim formulation of gravity.

We construct the action $S^{\prime}$ corresponding to the Hamiltonian (46). A new equality for the generalized velocity reads

$$
\partial_{0} A_{i}=\frac{\delta H^{\mathrm{gen}}}{\partial \pi_{i}}=\pi_{i}-\partial_{i} \lambda
$$

Expressing the generalized momentum $\pi_{i}$ from this equality and making the Legendre transform we find the required action

$$
S^{\prime}=\int d t \int d^{3} x\left(\frac{1}{2}\left(\partial_{0} A_{i}+\partial_{i} \lambda\right)\left(\partial_{0} A_{i}+\partial_{i} \lambda\right)-\frac{1}{4}\left(\partial_{i} A_{k}-\partial_{k} A_{i}\right)\left(\partial_{i} A_{k}-\partial_{k} A_{i}\right)\right) .
$$

The Lagrange factor $\lambda(x)$ related with the additionally imposed constraint appears in this action as a new independent variable. Denoting $A_{0}=-\lambda$ one easy recognizes in expression (48) the free electrodynamics action. The initial action (44) can be derived from it by fixing of the gauge $A_{0}=0$.

This example shows that the existence of additional first-class constraints in the canonical formalism can indicate that the initial theory without additional constraints is a result of fixing of gauge (probably partial) in some extended theory with an additional gauge symmetry. In particular, the initial embedding theory with action (3) having a four-parameter gauge group, where the independent variable is the embedding function, appears to be the result of the gauge fixing in Regge-Teitelboim formulation of gravity which has an eight-parameter gauge 
group and is described by the Hamiltonian (42). It should be noted that, as well known, the fixing of gauge in action usually leads to loss of some equations of motion. That is why the Regge-Teitelboim equations (4) have extra solutions.

The action of the extended theory corresponding to generalized Hamiltonian (42) of ReggeTeitelboim formulation of gravity was found in [10] in a way completely analogous to the one described in this section. It can be written in the form of the initial Einstein-Hilbert action

$$
S=\int d^{4} x \sqrt{-g^{\prime}} R\left(g^{\prime}\right),
$$

if we substitute for the metric $g_{\mu \nu}^{\prime}$ the modification of expression (2):

$$
g_{i k}^{\prime}=\stackrel{3}{g}_{i k}=\partial_{i} y^{a} \partial_{k} y_{a}, \quad g_{0 k}^{\prime}=\partial_{0} y^{a} \partial_{k} y_{a}-N_{k}, \quad g_{00}^{\prime}=N_{0}^{2}+g_{0 i}^{\prime} \stackrel{3}{g}^{i k} g_{0 k}^{\prime}
$$

(whence we obtain $g^{\prime 00}=\frac{1}{N_{0}^{2}}$; we note that these formulas differ from formulas in [10] by numeric factors because of changing of definition of constraint (14)). Here $N_{k}$ and $N_{0}$ are new independent variables (in addition to $y^{a}$ ), which are transformed into Lagrange multipliers in canonical formalism. The action (49) has an eight-parameter gauge symmetry, and the value $g_{\mu \nu}^{\prime}$ appears to be invariant under four of these eight transformations, which have constraints $\Psi^{A}$ as generators in the canonical formalism.

If we introduce a partial fixing of gauge by conditions

$$
N_{0}=\sqrt{\partial_{0} y^{a} \stackrel{3}{\perp}_{\perp a b} \partial_{0} y^{b}}, \quad N_{k}=0,
$$

then the quantity $g_{\mu \nu}^{\prime}$ coincides with the induced metric, and the action of the extended theory (49) transforms into the action (31) of the initial embedding theory. If we do not fix the gauge, then the quantity $g_{\mu \nu}^{\prime}$ still satisfies (in general case) the Einstein's equations. Therefore we can consider the quantity $g_{\mu \nu}^{\prime}$ to be the metric, which is invariant under additional symmetry transformation and coincides with the induced metric only in the mentioned gauge.

A disadvantage of the action (49) of the extended theory is the presence of a singled out direction of time related to the fact that the time and space components appear in formulas (50) in a different way. It would be interesting to find a modification of formulas (50) without singled out time direction but where the equations of motion still would be equivalent to the Einstein's equations.

Acknowledgments. The work was supported by the Russian Ministry of Education, Grant No. RNP.2.1.1/1575.

\section{References}

[1] Regge, T., Teitelboim, C.: General relativity à la string: a progress report. Proceedings of the First Marcel Grossmann Meeting, Trieste, Italy, 1975, ed. R. Ruffini, Amsterdam, North Holland, p 77 (1977)

[2] Deser, S., Pirani, F.A.E., Robinson, D.C.: Phys. Rev. D 143301 (1976)

[3] Tapia, V.: Clas. Quantum Gravity 6, L49 (1989)

[4] Franke, V.A., Tapia, V.: Nuovo Cimento B 107 (N 6), 611 (1992) 
[5] Capovilla, R., Escalante, A., Guven, J., Rojas, E.: Int. J. Theor. Phys. 48, 2486 (2009), arXiv:gr-qc/0603126

[6] Faddeev, L.D.: New action for the Hilbert-Einstein equations (2009), arXiv:0906.4639

[7] Faddeev, L.D.: New variables for the Einstein theory of gravitation (2009), arXiv:0911.0282

[8] Cordero, R., Molgado, A., Rojas, E.: Phys. Rev. D 79, 024024 (2009), arXiv:0901.1938

[9] Pavsic, M., Tapia, V.: Resource Letter on geometrical results for Embeddings and Branes (2000), arXiv:gr-qc/0010045

[10] Paston, S.A., Franke, V.A.: Theor. Math. Phys. 153 (N 2), 1581 (2007), arXiv:0711.0576

[11] Paston, S.A., Franke, V.A.: The gravity as a theory of embedding of space-time into the flat space of higher dimensions (in Russian). Proceedings of the 15 International V.A. Fock school for advances of physics 2005, ed. by V. Novozhilov, St.Petersburg, Publishing house of St.Petersburg State University, p 34 (2006)

[12] Gitman, D.M., Lavrov, P.M., Tyutin I.V.: J. Phys. A: Math. Gen. 23, 41 (1990).

[13] Arnowitt, R., Deser, S., Misner, C.: In: Witten, L. (ed.) Gravitation: An Introduction to Current Research. Wiley, New York (1962), arXiv:gr-qc/0405109 